\title{
LINEAR EXTENSIONS OF CONVEX SUBSETS
}

\author{
HIDEYUKI FUJIHIRA
}

\begin{abstract}
We give conditions for linear extensions of closed convex subsets of topological vector spaces to be closed.
\end{abstract}

1. Introduction. Let $A$ be a nonempty convex subset of a real topological vector space (t.v.s.), and let $A^{i}$ be the set of all points $x$ in $A$ which belong to the interior of each line segment through $x$ in $A$. The set $A^{i}$ is not the interior of $A$. Obviously $A^{i}$ is the radial kernel of $A$ in the linear manifold generated by $A$.

Let $A_{f}=A \backslash A^{i}$, and we call $A_{f}$ the frame of $A$. The structure of the frame is complicated. Even in a Banach space there may exist a bounded closed convex subset containing more than one point such that $A=A_{f}$. If a closed convex subset $A$ has the nonempty interior, then $A_{f}$ coincides with the boundary of $A$. But the frames of bounded closed convex subsets are, in many cases, dense in themselves.

2. Statement of the theorems. We begin with the following lemmas.

Lemma 1. Let $A$ be a nonempty bounded closed convex subset of a Hausdorff t.v.s. Then $A_{f} \neq \varnothing$ and $A=\operatorname{co} A_{f}$, where $\operatorname{co} A_{f}$ denotes the convex hull of $A_{f}$.

Proof. If $A$ consists simply of one point, then it is clear. Let $x$ be an element of $A$ and $t_{0}=\sup \{t>0: y+t(x-y) \in A\}$ for $y(y \neq x)$ in $A$. Since the intersection of $A$ and the line $L$ joining two points $x$ and $y$ is compact, we have $y+t_{0}(x-y) \in A_{f}$, and hence $A_{f} \neq \varnothing$. Since $x$ can be written as a convex combination of two extreme points of the line segment $L \cap A$ (both are the elements of $A_{f}$ ), it follows that $x \in \operatorname{co} A_{f}$. Therefore we obtain $A \subset \operatorname{co} A_{f}$, and it is clear that $A \supset \operatorname{co} A_{f}$. The proof is complete.

LEMma 2. Let $A$ be a closed convex subset of a t.v.s. with $0 \in A^{i}$ and $\Theta$ the linear extension of $A$. Then $A$ has nonempty interior in $Q$ if and only if $A_{f}$ is closed.

Proof. Since the radial kernel of $A$ is the interior of $A$ whenever $A$ has nonempty interior, it is clear that if $A$ has interior points in $Q$, then $A^{i}$ is open in $Q$, hence $A_{f}$ is closed.

Conversely, if $A_{f}$ is closed, then since $A^{i}$ is open in $A$, there exists a circled neighborhood $U_{0}$ of 0 such that $U_{0} \cap A \subset A^{i}$. Let $x$ be an element of $U_{0} \cap Q$. If $x \notin A^{i}$, then there exists an element $x_{f}$ of the intersection of $A_{f}$ and the line segment

Received by the editors January 12, 1983.

1980 Mathematics Subject Classification. Primary 52A05.

Key words and phrases. Convex sets, linear extensions, radial kernels, barrelled spaces. 
$[0, x]$. Since $U_{0}$ is circled, it follows that $[0, x] \subset U_{0}$ and, hence, $x_{f} \in U_{0} \cap A \subset A^{i}$. This is a contradiction and hence we obtain $U_{0} \cap \mathbb{Q} \subset A^{i}$. Then $A$ has nonempty interior in $Q$.

THEOREM 3. Let $A$ be a closed convex subset of a t.v.s. $V$ with $0 \in A^{i}$ and let $Q$ be the linear extension of $A$. If $A_{f}$ is closed, then $Q$ is closed. The converse is true if each closed subspace of $V$ is a barrelled space.

Proof. Let $\left\{x_{\alpha} ; \geqslant\right\}$ be a net in $\mathbb{Q}$ which converges to $x$. If $A_{f}$ is closed, then from Lemma 2, we can select a neighborhood, $U_{0}$, of 0 such that $U_{0} \cap \mathbb{Q} \subset A$. Let $V_{0}$ be a circled neighborhood of 0 such that $V_{0}+V_{0} \subset U_{0}$. Since $\left\{x_{\alpha}\right\}$ is a Cauchy net, there exists $\alpha_{0}$ such that

$$
\alpha \geqslant \alpha_{0} \Rightarrow x_{\alpha}-x_{\alpha_{0}} \in V_{0} .
$$

Therefore there exists $\lambda_{0}>0$ such that

$$
\alpha \geqslant \alpha_{0} \Rightarrow x_{\alpha} \in x_{\alpha_{0}}+V_{0} \subset \lambda_{0} V_{0}+V_{0} \subset\left(\lambda_{0}+1\right) U_{0},
$$

and this implies

$$
\alpha \geqslant \alpha_{0} \Rightarrow x_{\alpha} \in\left(\lambda_{0}+1\right)\left(U_{0} \cap \mathbb{Q}\right) \subset\left(\lambda_{0}+1\right) A .
$$

Since $\left(\lambda_{0}+1\right) A$ is closed, we obtain $x \in\left(\lambda_{0}+1\right) A \subset \mathbb{Q}$, and then $\mathbb{Q}$ is closed.

If the closed subspace $Q$ is a barrelled space, then $A$ has nonempty interior in $Q$ because $A \cap(-A)$ is a barrel in $\mathcal{Q}$. Therefore, by Lemma $2, A_{f}$ is closed and the proof is complete.

Theorem 3 implies that a linear extension $\mathcal{Q}$ of a compact convex subset $A$ with $0 \in A^{i}$ of a Banach space is closed if and only if $Q$ is finite dimensional.

The following corollary is a direct consequence of Theorem 3.

COROllary 4. Let $V$ be a locally convex Hausdorff t.v.s. and $Q$ a subspace of $V^{\prime}$, the dual of $V$. If there exists a bounded subset $M$ in $V$ such that $M^{\circ} \cap \mathbb{Q}$ is $\sigma\left(V^{\prime}, V\right)$-closed, then $Q$ is closed relative to the strong topology, where $M^{\circ}$ denotes the polar in $V^{\prime}$ of $M$. Moreover, if $V$ is semireflexive, then $Q$ is $\sigma\left(V^{\prime}, V\right)$-closed.

Proof. Since $M^{\circ}$ has nonempty interior with respect to the strong topology, $\left(M^{\circ} \cap Q\right)^{i} \neq \varnothing$. If $M^{\circ} \cap Q$ is $\sigma\left(V^{\prime}, V\right)$-closed, then it is strongly closed, and since it has nonempty interior in $\mathbb{Q}$ with respect to the strong topology, by Theorem $3, \mathbb{Q}$ is strongly closed.

If $V$ is a semireflexive space, then a strongly closed convex subset is $\sigma\left(V^{\prime}, V\right)$-closed, hence $Q$ is $\sigma\left(V^{\prime}, V\right)$-closed.

THEOREM 5. Let $V$ be a Hausdorff barrelled space and $A$ a bounded closed convex subset of $V$ with $0 \in A^{i}$. If the codimension of the linear extension $Q$ of $A$ is finite, then $\mathbb{Q}$ is closed.

Proof. We prove the theorem for codimension one. The other case can be proved similarly. Let $y \neq 0$ be an element of the algebraic complementary subspace to $Q$ in $V$. Let $B=\operatorname{co}(A \cup\{y\})$. Then $B$ is closed. Each $z$ in $V$ can be written as 
$z=\alpha y+\lambda a\left(\alpha \in R, \lambda>0, a \in A^{i}\right)$. Since the line segment $[t a, \beta y]$, for $0<t<1$ and $0<\beta<1$, is contained by $B$, we obtain that, for sufficiently small $t>0$,

$$
t_{0} y+t\left(z-t_{0} y\right)=\left(t_{0}+t\left(\alpha-t_{0}\right)\right) y+t \lambda a \in B \quad\left(0<t_{0}<1\right) .
$$

Therefore, for $0<t_{0}<1, t_{0} y$ is an element of the radial kernel of $B$ in $V$, and hence $B^{i} \neq \varnothing$. Therefore $B$ has interior points. Let $x_{0}$ be an interior point of the line segment $[0, y]$. From Theorem 3, this theorem will follow if it is proved that $\left(B-x_{0}\right) \cap \mathbb{Q} \subset A$.

Let $z=\lambda a+(1-\lambda) y(0 \leqslant \lambda \leqslant 1, a \in A)$ be an element of $B$. Since $x_{0}$ can be expressed as $x_{0}=\lambda_{0} y\left(0<\lambda_{0}<1\right)$, if $z-x_{0} \in \mathcal{Q}$, then $\lambda=1-\lambda_{0}$. Hence we obtain $z-x_{0}=\left(1-\lambda_{0}\right) a \in A$, and this establishes the desired result.

\section{REFERENCES}

1. H. H. Schaefer, Topological vector spaces, Macmillan, New York, 1967.

2. J. L. Kelley, I. Namioka et al., Linear topological spaces, Van Nostrand, Princeton, N. J., 1963.

3. N. Bourbaki, Éléments de mathématique, Espaces vectoriels topologiques, Chap. 3-5, Hermann, Paris, $1955^{\circ}$.

Department of Mathematics, University of UtSunomiya, Minemachi, Utsunomiya, Japan 